\title{
Transient Osteoporosis of Hip in A Middle-Aged Man
}

\author{
Gaurav Sharma*1, Akshay Bhardwaj ${ }^{2}$, Nisha Relwani ${ }^{3}$ and Shubhangi Dubey ${ }^{4}$ \\ ${ }^{1}$ Department of Orthopaedics, Prakash Institute of Medical sciences, India
}

${ }^{2} D N B$ Orthopedics, DDU Hospital, India

${ }^{3}$ Assistant Professor, MGM Medical College, India

${ }^{4}$ MBBS, Babasaheb Ambedkar Hospital, India

Received: October 27, 2017; Published: November 02, 2017

*Corresponding author: Gaurav Sharma, MS Orthopaedics, Assistant Professor, Department of Orthopaedics, Prakash Institute of Medical sciences, Islampu-Sangle road, Tal- Walwa, Sangli, Uran, Islampur- 415409, Maharsahtra, India, Tel: 9545455852; Email: sharmagaurav@live.com

\begin{abstract}
Introduction: Transient Osteoporosis of Hip is a benign, acute onset, self-limiting disorder of unknown aetiology commonly seen in middle aged men and pregnant females.

Case Report: We report a case of acute onset sharp pain in right hip in a physician without history of antecedent trauma. The diagnosis of Transient Osteoporosis of Right Hip was made based on clinical and MRI findings. The probable cause can be attributed to long standing working condition. There was complete resolution of symptoms by conservative treatment in the form of analgesics and non-weight bearing.

Conclusion: High level of suspicion should be kept in mind when dealing with patients with sudden onset non-traumatic joint pain to avoid unnecessary investigations and surgeries.
\end{abstract}

Keywords: Osteoporosis; Arthralgia; Hip; Acute pain

\section{Introduction}

Transient Osteoporosis of Hip (TOH) is an acute, idiopathic, benign, self-limiting entity, more commonly seen in middle-aged men and women in third trimester of pregnancy or immediate post-partum period [1-4]. This disorder of unknown aetiology has a sudden onset, usually without any antecedent history of trauma or injury. We hereby report a case of Transient osteoporosis of hip affecting a middle aged male physician.

\section{Case Report}

A 38 year old physician reported with sudden onset pain in right hip joint since 2 weeks. The pain was severe sharp sudden in onset, increased with activities like walking, standing for longer duration, getting up from bed and relieved completely on rest. Being a physician himself, he first neglected the pain and went to a physiotherapist to try local modalities with limited relief. There was no history to trauma/injury to right hip, no history of morning stiffness, anorexia, fever, weight loss, night cries, skin rashes or fever. There was no history of alcohol consumption or smoking. On examination, the patient was walking with an antalgic gait. The local temperature and the skin condition over right hip were normal. The affected hip had generalized tenderness with terminal restriction of movements. His haematological investigations revealed a haemoglobin of $12.3 \mathrm{gm} \%$, Total leukocyte count of $5800 / \mathrm{mm}^{3}$, ESR of $32 \mathrm{~mm}$ at the end of one hour, all of which were within normal limits.

Initial radiographs in form of Pelvis with both hips Antero posterior view and frog leg lateral view revealed no bony abnormality with maintained joint space. His synovial aspiration was normal in appearance and was negative for acid fast bacilli, gram staining and was sterile with no growth on culture and sensitivity. With no conclusive diagnosis, MRI of right hip joint was done which showed low intensity signals of T1 weighted image and a high intensity on T2 weighted image covering the femoral head, neck and extending to the intertrochanteric region (Figure 1). Thus, with the aforementioned classical features, a diagnosis of Transient Osteoporosis of right Hip was made. The conservative treatment was started in the form of non-steroidal inflammatory drugs was started. The patient responded drastically to the conservative treatment within 3 weeks from the initiation of treatment. Regular monthly follow-up till 6 months followed by bi annual and annual follow-up till 2 years was done. The patient showed no signs of recurrence or worsening of the symptoms and was completely normal (Figure 2). 


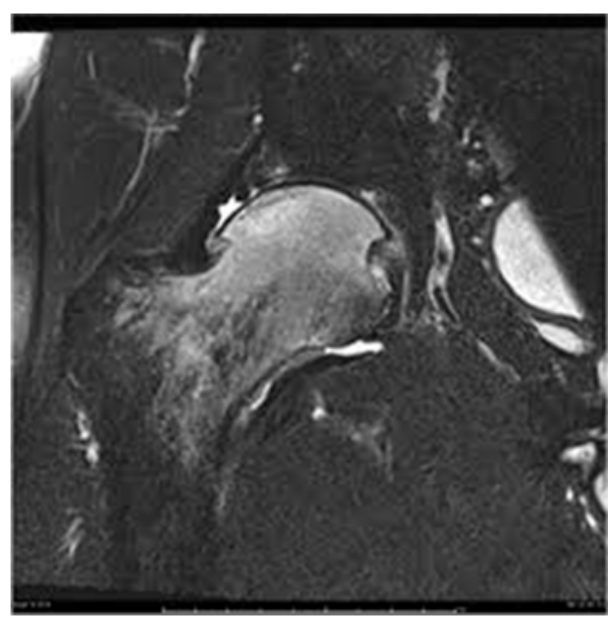

Figure 1: Transient Osteoporosis of Right Hip.

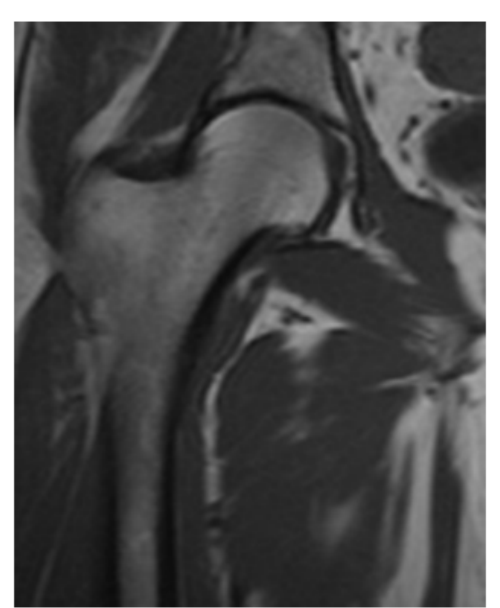

Figure 2: Complete Resolution at follow-up.

\section{Discussion}

Ever since Curtiss and Kincaid [5] described this entity among 3 pregnant females as transitory demineralization of the hip, this term has been a topic of interest due to its unknown aetiology. Albeit, the most commonly affected joint is Hip, other joints such as knee, foot and ankle has not been an exception6.

Various hypothesis and causes have been stated as the plausible explanation for this disease namely the neurogenic hypothesis, viral infection, disuse osteopenia, non-traumatic reflex sympathetic dystrophy and localized ischaemia, but none has proved to be conclusive [6,7]. However, women with decreased bone mass and parathyroid hormone related protein in pregnant females especially in the later trimester has been proved to be a cause for this disease [8]. Hadidy et al. [9] in their retrospective study of 17 patients in a span of 7 years, reported a higher incidence of TOH as compared to osteonecrosis and postulated it to be due to long hours of standing. There was no possible cause of TOH in the present case. Being a physician, long standing can be a possible cause. Scharpira [10] in their study mentioned that left hip is more commonly involved and that the male to female ratio is $3: 1$. He also identified three distinctive phases of TOH.
Initial rapid aggravating phase characterized by intense pain which increases on weight bearing with functional impairment during the first month after the onset of symptoms. This phase remains for another 1-2 months and later progresses to the second phase where there is demineralization of the femoral head, neck and the intertrochanteric region without affecting the joint space on plain radiograph. Magnetic resonance imaging serves to be an investigation of choice with high sensitivity in this stage with non-homogenous marrow involving the mentioned areas. The final spontaneous regression stage usually lasts for about 3-4 month where there is complete resolution of clinical as well as radiological features. The diagnosis of TOH on radiography is often late but typical with periarticular diffuse osteopenia affecting the femoral head, neck and even the intertrochanteric region. It rarely affects the pelvis and the joint space even in advanced stages which differentiates it from osteonecrosis. There is decreased joint space in osteonecrosis with involvement of the anterosuperior region of the femoral head and sparing the neck and trochanter. Bloem [11] described the MR finding in TOH as ill-defined area of decreased signal intensity is seen on T1 weighted images, with an area of increased signal intensity on $\mathrm{T} 2$ images suggestive of bone marrow edema.

In the present case, MR imaging led to a conclusive diagnosis of TOH. Radionuclide scan can be beneficial with diffuse and homogenous uptake initially with a gradual decrease in late stages. It has high sensitivity but low specificity [7]. No Scintigraphy scan was performed for the present case. Differential diagnosis of rheumatoid arthritis, infective arthritis, monoarticular conditions like idiopathic chondromatosis and pigmented villonodular synovitis, osteoarthritis, primary/secondary bone tumour and tuberculosis of the bone should be kept in mind while dealing with the patients with non-traumatic hip pain. A possibility of reflex sympathetic dystrophy (RSD) should also be kept in mind. However, RSD is associated with history of re-surgery, sensory disturbances with trophic changes with a predilection toward small bones and different MRI pattern than TOH. While it is difficult to differentiate TOH from osteonecrosis of the femoral head in the early stages, careful clinical assessment can help to rule out the diagnosis. Apart from acute onset pain and antalgic gait, patients with osteonecrosis have resting pain, differential rotation positive and painful range of movements. Radiologically, the lesion is typically confined to anterosuperior aspect of the femoral head with joint space narrowing and radiolucent areas with sclerosis in the head sparing the neck and trochanter region. Furthermore, MRI in osteonecrosis shows segmental well demarcated lesion in the femoral head with double line sign which is a pathognomic feature.

Various treatment options have been proposed for TOH with conservative methods being the mainstay of the same. Nonweight bearing with a course of non-steroidal anti-inflammatory medications and assurance is required I almost all the cases. Oral and intra-articular steroid therapy, calcitonin and sympathetic blocks have been proposed by few with limited results [7]. Medical management with different forms of oral and intravenous 
bisphosphonates like alendronate, pamidronate, ibandronate and zolebdronate have been tried by few in the past with good results [12-14]. Bisphosphonates reduces the increased bone destructive activities leading to restoration of the bone mineralization and decrease in pain dramatically. Although it provides symptomatic relief, it does not change the course of the disease. No form of bisphosphonates was used in the present case. Hoffman et al. [15] and Guerra et al. [16] in their studies have stressed upon surgical intervention using core decompression in patients with severe pain and in cases which do not respond to the conservative treatment.

\section{Conclusion}

Transient Osteoporosis of Hip should always be kept as a differential in cases with acute onset pain in joint in middle-aged males. Observation, reassurance, non-weight bearing and nonsteroidal anti-inflammatory drugs remain the mainstay of this self-limiting condition. High level of suspicion can help to avoid unnecessary investigations and surgeries.

\section{References}

1. Korompilias AV, Karantanas AH, Lykissas MG, Beris AE (2008) Transient Osteoporosis. J Am Acad Orthop Surg 16(8): 480-489.

2. Pande K, Aung TT, Leong JF, Bickle I (2017) Transient Osteoporosis of the Hip: A Case Report. Malaysian Orthopaedic Journal 17(1): 77-78.

3. Gupta P, Sharma S, Gupta V, Singh D, Agarwal A, et al. (2005) Transient Osteoporosis of Hip- A Case Report. Indian Journal of Orthopaedics 39(4): 257-259.

4. Van Wagenen K, Pritchard P, Taylor JA (2013) Transient osteoporosis of the hip: A Case report. J Can Chiropr Assoc 57(2): 116-122.

5. Curtiss PH, Kincaid WE (2959) Transitory demineralization of the hip in pregnancy. J Bone Joint Surg (Am) 41-A: 1327-1333.
6. Lakhanpal S, Ginsburg WW, Luthra HS, Hunder GG (1987) Transient regional osteoporosis. A study of 56 cases and review of literature. Ann Int Med 106(3): 444-450.

7. Crespo E, Sala D, Crespo R, Silvestre (2001) Transient Osteoporosis. Acta Orthopaedica Belgica. 67(4): 330-337.

8. Szwedowski D, Nitek Z, Walecki J (2014) Evaluation of transient osteoporosis of the hip in magnetic resonance imaging. Pol j radiol 79: 36-38.

9. Hadidy AM, Al Ryalat NT, Hadidi ST, Tarawnech ES, Hadidi MT, et al. (2009) Male transient hip osteoporosis: are physicians at a higher risk? Arch Osteoporos 4(1-2): 41-45.

10. Scharpira D (1992) Transient osteoporosis of the hip. Sem. Arthr. Rheumat 22(2): 98-105.

11. Bloem JL (1998) Transient osteoporosis of the hip. MR imaging. Radiology 167(3): 753-755.

12. Miltner O, Niedhart C, Piroth W, Weber M, Siebert CH (2003) Transient osteoporosis of the navicular bone in a runner. Arch Orthop Trauma Surg 123(9): 505-508.

13. Ringe JD, Dorst A, Faber H (2005) Effective and rapid treatment of painful localized transient osteoporosis (bone marrow edema) with intravenous ibandronate. Osteoporosis Int 16(2): 2063-2068.

14. Seok H, Kim YT, Kim SH, Cha JG (2011) Treatment of transient osteoporosis of the hip with intravenous zolendronate-A case report. Ann Rehabil Med 35(3): 432-435.

15. Hofman S, Schneider W, Breitenseher M, Urban M, Plenk H (2000) Transient osteoporosis as a special reversible form of femur head necrosis 29(5): 411-419.

16. Gurre JJ, Steinberg ME (1995) Distinguishing transient osteoporosis from avascular necrosis of the hip. J Bone Joint Surg (Am) 77(4): 616 624.

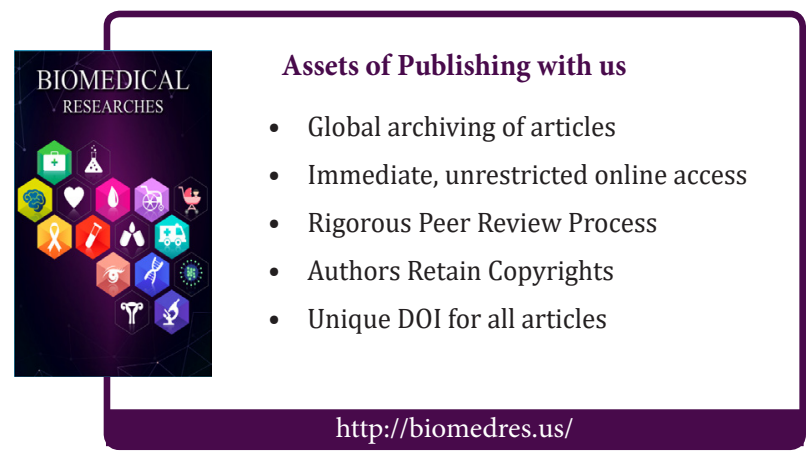

